\title{
NEW CONCEPT DEVELOPMENT MODEL: EXPLORATIVE STUDY OF ITS USABILITY IN INTELLIGENCE AUGMENTATION
}

\author{
Irena Drašković, Maikel Couwenberg \\ RUNMC, Radboud University, Nijmegen, The Netherlands \\ I.Draskovic@iq.umcn.nl, \\ Janos Sarbo \\ ICIS, Radboud University, Nijmegen, The Netherlands \\ janos@cs.ru.nl
}

\begin{abstract}
Keywords: Intelligence augmentation, problem solving, knowledge representation, conceptual representation and interpretation, empirical study.

Abstract: Intelligence augmentation (IA) is used in for improving the efficiency of human problem solving by making use of computer support. To make it work, we need a 'human-compatible' formal model of knowledge representation. Here we report on empirical test of one such model. The results show that the model can be used to analyse concept formation in human problem solving. This means that the model is congruent with human information processing system. Being a formal model, it can be used in a wide range of IAapplications such as computer supported tutoring systems and human-computer interfaces.
\end{abstract}

\section{INTRODUCTION}

The main goal of research on Intelligence Augmentation (IA) is to develop tools and methods to support and improve the effectiveness of human intelligence in solving complex problems. However, on one side, we do not have a full understanding of human cognition and, on the other side, we do not yet have at our disposal complex parsers to interface with different informal representations employed in human processing. According to Douglas C. Engelbart (1962), the aim of IA tools is to optimize sensory, motor, and cognitive human capacities. To achieve better efficiency and effectiveness of computer systems for knowledge representation, 'human-compatible' formal models of knowledge representation are needed. In the present paper, we report on an empirical study exploring the features of such a formal model of human information processing.

Recent empirical studies on human processing suggest that e.g., syntactic and semantic information processing is quasi-simultaneous (Hagoort, Hald, Bastiaansen, \& Petersson, 2004). This renders translation between these representational formats improbable. In addition, it underscores the importance of developing a uniform knowledge representation model in other modalities as well. As far as we know, current computational models of knowledge representation do not meet the demand of a uniform representational format and interpretation processes across different kinds and modalities of information (Shadbolt, Hall \& Berners-Lee, 2006). Rather, those models use different representational formats for the different knowledge domains. From the computation point of view, translation between different formats is inefficient. Moreover, the development of translator programs is non-trivial (Aho \& Ullman. 1972).

The present paper introduces a uniform knowledge representation model together with a preliminary testing of its validity. So far, the usability of this model has been tested in the following knowledge domains: (morpho-)syntax, naïve logic, reasoning, and mathematics (Sarbo, Farkas \& Bremen, 2006). In the present paper we explore correspondence between the predicted and 
the observed output, in different information processing stages specified by our model.

\subsection{Model description}

\subsubsection{Theoretical background}

In developing our model, we considered developmental aspects of human cognition. According to the Social Constructivist model of Piaget, learning and cognitive processes are adaptive 'tools' aiding human interaction with the surrounding. Focusing on cognitive development, Piaget defined different stages in child development (Rigter, 1996):

- $\quad$ Sensory-motor phase. Objects and object characteristics are learned and recognized through perception and motor manipulation; this knowledge is stored as concepts or abstract representations of object characteristics (e.g., sweet).

- Pre-operational phase. Percepts are explained through reasoning. It is assumed that the most salient concept properties are included into interpretations.

- Concrete operational phase. Learning that different points of view are possible.

- Formal operational phase. Reasoning without preceding perception, development of abstract reasoning and hypothesizing (Delfos, 2000).

J.S. Bruner (e.g., 1966) describes cognition as a cyclic process including hypothesizing, informative, and confirmative phases. Similar to Piaget, Bruner distinguishes 3 phases in child cognitive development:

- $\quad$ Enactive phase. Learning through physical contact with the surrounding.

- Iconic phase. Decisions based on sensorymotor perception.

- Symbolic phase. Meaning construction through symbolic interpretation of information.

We will assume that a 'fully developed' cognitive system incorporates an amalgam of processes and operations specified by Piaget and Bruner and that these are effective in concept formation. Our model extends these notions and builds a bridge towards IA.

\subsubsection{The model}

In Farkas \& Sarbo (2000) and, more recently, in Breemen \& Sarbo (2009), a novel formal conceptualization model is presented (see Figure 1). Conceptualisation is described through 3 processing stages implementing 3 kinds of operations, ranging from perceptual analysis (analysis of sensory input) to meaningful response, such as predication or motor response. For each stage, input and output are specified alongside the concept types. The model is interactive in that it takes into account both the internal context as well as the stimulus characteristics.

Stage 1- PERCEPTION: Sensory stimulus triggers perceptual analysis and sorting; further processing proceeds in two processing paths:

1. A, B: processing of the 'raw' perceptual data (A objects in focus, $\mathrm{B}$ - events in the world or model associated with these objects); a distinction is made between the background and the stimulus;

2. $\neg \mathrm{A}, \neg \mathrm{B}$ (not A, not B): activation of knowledge on both the objects in focus and the associated events (which are not(objects), and not(events) by themselves).

Output of the sorting operation performed in this stage is identification of the important components in the input and activation of knowledge on these components. This stage is a 'transition' from the 'world' to a 'model' of the world. Hereby the notion of contrast is important: perception is 'perception of contrast/change'.

Stage 2 - CONTEXTUALIZATION: Output from stage 1 is abstracted into types through matching with existing prototype concepts (Smith, Osherson, Rips \& Keane, 1988). Contextualization incorporates: lexical access of the perceived qualities (qualia) as types independent from each other; completion of concept types (qualities) from the previous stage by using internal context or knowledge of the world per quality; matching of the input with internal context. By sufficient correspondence and context to relate the 2 types of qualities with each other, the next stage is entered. This stage is comparable to lexical access and semantic interpretation (Margolis \& Laurence, 1999).

Stage 3 - PREDICATION: Complements from the two processing paths are related to each other. The most plausible relation between the qualities is established and the qualities are put together into a proposition expressing a hypothesis on the state of the world. This stage includes testing of the hypothesis with relevant sensory information whereupon a new cycle may start. If the hypothesis is disconfirmed, either new complementation context is searched for, or other focus is taken (cf. polysemy - 'bank').

It is plausible to assume that with complex problems the above outlined conceptualization 
process is recursively used whereby the propositions formed at the end of one cycle serve as input for the next cycle. Per cycle, one proposition is generated. A cycle is delimited by identification (naming) of a relation (e.g., 'Square A is larger than square B').

The process is goal driven with the goal being the formulation of a proposition. In solving a problem, the number of embedded analyses (cf. recursion) can be affected by three parameters:

1. What is in focus (always a contiguous segment of input qualities).

2. Input complexity (number of propositions that exhaustively describe a phenomenon in focus).

3. Internal context (relevant knowledge of the world).

These are the sources of inter- and intra variability in interpretation. By a well defined problem (see Plato, 380 B.C.) with a generally accepted solution, it is possible to determine in advance the goal governing the entire conceptualization process. Exploiting the thinkingout-loud method in the process of solving a complex mathematical problem, it is possible to gather verbal reports containing utterances reflecting the interpretation process. Utterances can be coded as types of concepts. The degree of match with the concept types specified by our model can be determined.

\subsection{Research question and hypothesis}

According to our model, concepts are formed through 3 development stages. In each stage different types of concepts or signs are generated. We assume that the stages are sequentially ordered. Advance to the next stage is determined by completion of the previous stage. Generated concepts are expected to be classifiable into concept types specified by our model. The following research question will be investigated: Can verbal utterances produced during the solution of an abstract task be classified into concept types specified by our model?

Hypothesis: Our model specifies 3 processing stages: perception, contextualization, and predication, each having specific input and output. The model assumes an ordering of the processing stages; output of the earlier stages serves as input for the later stages. We assume that knowing the output i.e., verbal reports, can be used to infer the underlying processing stages. In coding the verbal utterances into concept types specified by our model, we use the terminology from a Peircean interpretation of our model (Peirce, 1931) as shown in Figure 2 (see also, Breemen \& Sarbo, 2009). Below, these are given in small caps.

\section{Stage 1 - Perception:}

- signal or external trigger as qualia (QUALISIGN);

- spatial and temporal localization of the signal (ICON, SINSIGN);

- knowledge on the signal (INDEX).

Stage 2 - Contextualization:

- prototype knowledge on A (RHEME);

- prototype knowledge on B (LEGISIGN);

- prototypes in context (DICENT, SYMBOL).

\section{Stage 3 - Predication output:}

- concepts in a relation (ARGUMENT).

In order to test the hypothesis, we made use of a complex task and a problem which is largely unknown to the participants but which they should be able to solve according to their developmental stage, prior knowledge and familiarity with similar tasks. In order to minimize variability in prior knowledge a homogeneous group of participants will be selected, namely primary school grade 8 pupils (age: $11-12$ years). These pupils are normally not familiar with the specific problem (see section 2.2) and the specific mathematical reasoning needed to solve this problem. However, they do possess sufficient knowledge to be able to perform the task. The hypothesis was tested in an experiment described below. Dependent variable is percentage of concepts produced in order congruent with our model.

\section{METHOD}

To test our hypothesis we made use of a method in which participants solve a difficult geometrical problem while thinking-out loud. Their verbalizations were recorded and transcribed. Subsequently, verbal utterances were coded into the above specified nine output concepts. The use and the order of the three above described processing stages were determined on the basis of the prevailing 'output concepts'. This way, we were able to determine whether the observed conceptualization unfolds according to the stages specified by our model. This method is rather coarse in that verbalizations need not be entirely synchronous with the actual cognitive processing. We assume that the nine types of output concepts are 'tied' to the respective processing stages of Perception, 
Contextualization and Predication (e.g., qualisign, 'icon' etc. - Perception; 'rheme', 'dicent', etc. Contextualization).

\subsection{Participants}

Twenty-eight 8th graders from the primary school in Nuland, The Netherlands, took part in this experiment. Age range was 11-12 years. The participants were assumed not to be familiar with the problem since it is not a part of their Math course. This was confirmed by the teachers who reported that knowledge directly needed to solve this problem individually has not been acquired. This sample was chosen because many IA applications are targeting the respective population. Regarding their cognitive development, 8th graders are similar to the adults (Delfos, 2000).

\subsection{Task}

Participants were presented a problem based on Plato's Meno (Plato, 1871). They were shown a picture of a square and were to find out how to determine the length of the sides of another square which is half as large as the first one. We chose this problem because its solution is straight forward, as outlined in (Magnani, 2001). At the same time the problem is complex enough to elicit sufficient amount of verbal utterances to be analyzed, as determined in a pilot study involving two participants. The participants rated the problem as difficult although at their school level they have already learned to compute the area of geometrical figures which is necessary to solve this problem.

\subsection{Procedure and materials}

The experiment was conducted using a standard protocol. All sessions were videotaped. The time intervals needed to solve the problem were registered by the experimenter using a stop watch. The setting was an empty classroom; a familiar work surrounding for 8th graders. The experiment was conducted individually. The experimenter was seated in an L-setting with respect to the pupil in order to avoid a suggestion of a 'leadership role' to the experimenter since this may affect the pupils' level of commitment to solving the problem. The experiment was conducted during regular school time. The experimenter was instructed not to interfere with the process of solving the problem unless this is indicated in the protocol. Each session started with experimenter giving an instruction about the task and the procedure. The recordings contained on average 75 verbal utterances.
Instruction: "First of all you will receive a card with a drawing on it. The drawing 'represents' a geometrical problem. Your task is to uncover the problem and to find its solution. While doing this I would like you to say loudly everything you're thinking about this problem."

This is called 'thinking-out-loud' method. Subsequently, the participants were handed over the card with the drawing representing the Meno problem (see Figure 3) and the session started. It was determined in advance in which situations the experimenter will interfere and how: If a participant was stuck with a (part of) the problem (operationalized as inactive for 20 seconds) or if he/she made a mistake, the experimenter prompted him/her to try again and solve the problem or to try and correct the error. Few types of errors were anticipated upon which were already described in Plato's Meno and which also occurred in the pilot study. Additional material was developed to facilitate the problem solving by providing clues to shift the participants' focus in problem interpretation. This material was provided if needed in 3 different orders assigned randomly. An illustration of additional material is given in Figure 3.

Coding procedure. All transcribed verbal utterances were first assessed for their contribution to the solution of the Meno problem. Two kinds of codes were assigned: 1. contributes to the solution of the problem; and 2. 'side-tracking' or 'errors' like wrong perception/representation/interpretation of the problem, wrong assumptions, and logical errors. For the former kind of utterances a coding system was developed with types of concepts and examples specified.

Examples coding of the verbal utterances:

1. Prototype knowledge on a stimulus (RHEME) "like a square."

2. Prototypes in context (DICENT, Symbol) - "The square on the left side.."

3. Concepts in a relation (ARgument) - "This square is twice as big as the other one."

In order to validate the coding system two experts independently coded a sample of verbal protocols.

The degree in which the conceptualization process in solving the Meno problem is congruent with the conceptualization process as specified by our model was determined on the basis of prevalence of 'correctly' formed 'argument' concept types i.e., 'arguments' preceded by concept types from any of 
the preceding processing stages in order specified by our model.

\subsection{Analyses}

The inter-rater reliability of the coding system was determined using Cohen's Kappa, and means and standard deviations (SD) were computed using Statistical Package for the Social Sciences, version 14 (SPSS 14).

\section{RESULTS}

The inter-rater reliability for the coding criteria was high (Cohen's Kappa = 0,924). In total, 1690 verbal utterances were coded. Average percentage of task related utterances was $\mathrm{M}=79 \%$ ( $\mathrm{SD}=17.07)$. Average percentage of utterances classifiable into our concept types was $84(\mathrm{SD}=10.5)$. Average percentage of congruent 'arguments' was 42 $(\mathrm{SD}=37.81)$.

\section{DISCUSSION}

Our preliminary results show high level of congruence of concepts comprising verbal reports with the concept types specified in our model. Moreover, also the order of concept formation as inferred from verbal reports is congruent with the order of processing stages specified in our model.

Human conceptualization can be fast. In order to get hold of the unfolding interpretation process we introduced a task that, by virtue of its complexity, forces problem solving to be split into stages. In the first stage, subjects are typically stuck at a trivial interpretation of their input (e.g., 'There is a mathematical problem'). In this stage, concept types from the lower part of the model schema (Figure 1) are dominantly produced. Further stages are more difficult and often re-focussing is needed in order to proceed in solving the problem. Alongside, concept types from higher levels of the model schema are generated (see Figure 1). Note that additional material provided to participants only served the purpose of shifting attention, thus enabling emergence of alternative interpretations of the problem, rather than offering information necessary to solve the problem. The findings suggest that solving the problem is effective if the interpretation process proceeds as suggested in our model.

\section{CONCLUSIONS}

We have shown that our model can be used to analyse concept formation in human problem solving. This means that the model is highly congruent with human information processing. We believe that our formal model can contribute to a further development of existing IA-applications such as computer supported instruction and humancomputer interfacing as it may serve as an explicit basis for building such applications. Computer assisted studies reviewed in Seo \& Bryant (2009) did not show conclusive effectiveness despite the relatively large effect sizes obtained in these studies. However, more experiments tapping into on-line processing are needed in order to explore the features of the model more extensively.

\section{REFERENCES}

Aho, A.V., Ullman, J.D., 1972. The Theory of Parsing, Translation and Compiling, vol. 1, Prentice Hall.

Breemen, A.J.J.van, Sarbo, J.J., 2009. The machine in the ghost: The syntax of mind, Signs - International Journal of Semiotics, Royal School of Library and Information Science. Denmark.

Bruner, J.S., 1966. Toward a Theory of Instruction, Harvard University Press.

Delfos, M.F., 2000. Luister je wel naar míj? Gespreksvoering met kinderen tussen vier en twaalf jaar, Uitgeverij SWP, Amsterdam.

Engelbart, D.C., 1962. Augmenting Human Intellect: A Conceptual Framework, AFOSR-3233, Stanford Research Institute, Menlo Park, CA.

Farkas, J.I., Sarbo, J.J., 2000. A Logical Ontology. In: ICCS'2000. Shaker Verlag. Darmstadt (Germany).

Hagoort, P., Hald, L., Bastiaansen, M., Petersson, K-M., 2004. Integration of word meaning and world knowledge in language comprehension, Science, vol. 304.

Margolis, E. and Laurence, S. (Eds.). 1999. Concepts: Core Readings. M.I.T. Press.

Magnani, L., 2001, Abduction, Reason, and Science, Kluwer Academic/Plenum Publisher, New York.

Peirce, C. S., 1931-58, The Collected Papers of C. S. Peirce, vols. 1-6, ed. Charles Hartshorne and Paul Weiss; vols. 7-8, ed. A. W. Burks, Cambridge: Harvard.

Plato, 1871, Meno, Translation of Benjamin Jowett, web edition at http://classics.mit.edu/Plato/meno.html

Rigter, J., 1996. Het palet van de psychologie, Stromingen en hun toepassingen in de hulpverlening, Uitgeverij Coutinho, Bussum (NL). 
Sarbo, J.J., Farkas, J.I., Breemen, A.J.J.van, 2006. Natural Grammar. In: Semiotics and Intelligent System Development. Idea Group Publishing. Hersey (PA).

Shadbolt, N., Hall, W., \& Berners-Lee, T., 2006. The

Semantic Web Revisited. IEEE INTELLIGENT SYSTEMS.

Smith, E., Osherson, D., Rips, L., Keans, M. 1988. Combining prototypes: a selective modification model, Cognitive Science, 12.

Seo, Y.J., Bryant D.P., 2009, Analysis of studies of the effects of computer-assisted instruction on the mathematics performance of students with learning disabilities, Computers \& education, 53(3), 913-928.

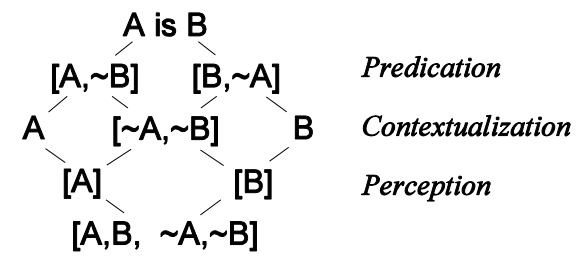

Figure 1. Model of development of concept types.

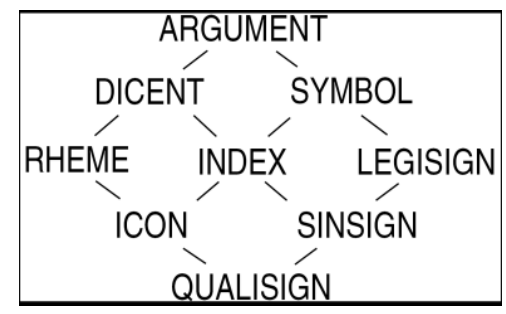

Figure 2. Concept type in problem solving.
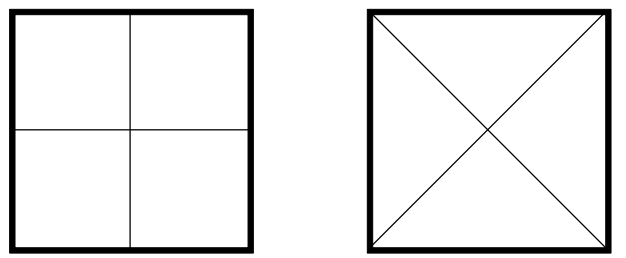

Figure 3. On the left side of the figure participants see a square for which the method of computing the length of the sides has to be determined. In those cases in which participants were stuck with the problem, the square on the right side of this figure was provided showing an alternative way of slicing the square which allows for refocusing and advancement to the solution. 